\title{
Factores asociados a la duración del hemofiltro en técnicas continuas de depuración extracorpórea en el paciente ingresado en cuidados intensivos
}

\author{
Francisco Javier Berrocal-Tomé1,2, Mónica Maqueda-Palau ${ }^{1,3}$, Cristina Moreno-Jiménez ${ }^{1}$, Ainoha de Dios-Guerra ${ }^{1}$ \\ ${ }^{1}$ Unidad de Cuidados Intensivos. Hospital Universitari Son Espases. Palma. España \\ ${ }^{2}$ Grupo de trabajo en Técnicas Extracorpóreas de la Sociedad Española de Enfermería Intensiva y Unidades \\ Coronarias (SEEIUC) \\ ${ }^{3}$ Grup d’Investigació en Cures. Cronicitat i evidències en salut (CurES-IdISBa). España
}

Como citar este artículo:

Berrocal-Tomé FJ, Maqueda-Palau M, Moreno-Jiménez C, De Dios-Guerra A. Factores asociados a la duración del hemofiltro en técnicas continuas de depuración extracorpórea en el paciente ingresado en UCI. Enferm Nefrol. 2021 Oct-Dic;24(4):389-97

\section{Resumen}

Objetivos: Determinar la vida media de los hemofiltros en el paciente crítico ingresado en la unidad de cuidados intensivos y los principales factores asociados a su duración.

Metodología: Estudio descriptivo observacional transversal, realizado en una Unidad de Cuidados Intensivos de adultos polivalente. Se estudiaron los hemofiltros colocados en 67 pacientes mayores de 18 años, entre enero y noviembre de 2019. Variables: edad, sexo, peso, unidad de ingreso, velocidad de flujo sanguíneo, fracción de filtrado, débito horario, anticoagulación del sistema, tiempo de tromboplastina activada (TTPA), indicación médica de la terapia, causa de la retirada, localización del catéter, hora de inicio y finalización de la terapia.

Resultados: La edad media de los pacientes fue de 62,66 años $( \pm 9,95), 81(71,64 \%)$ hombres. Se analizaron un total de 238 hemofiltros con una vida media de 26,28 horas $( \pm 22,8)$. El $80,1 \%$ de los catéteres fueron femorales, el $19 \%$ yugulares y el $0,8 \%$ subclavios. Se empleó como terapia de anticoagu-

\section{Correspondencia:}

Francisco Javier Berrocal Tomé

E-mail: javierbtya@gmail.com; franciscoj.berrocal@ssib.es lación, heparina sódica en un $45,8 \%$, citratos en el $20,2 \%$ y en un $34 \%$ no se utilizó anticoagulación. La velocidad media de flujo sanguíneo fue de 190,08 ml/ min $( \pm 53,48)$. Se encontró relación estadística entre las variables flujo sanguíneo ( $r s=0,208 ; p=0,001)$, localización del catéter y duración del hemofiltro $(p=0,03)$.

Conclusiones: La vida media del hemofiltro fue de 26 horas. La velocidad del flujo sanguíneo y localización del catéter son factores que repercuten en la duración del hemofiltro.

PALABRAS CLAVE: hemofiltración; cuidados críticos; anticoagulantes; dispositivo de acceso vascular; velocidad del flujo sanguíneo.

Factors associated with haemofilter duration in continuous extracorporeal clearance techniques in intensive care unit patients

\section{Abstract}

Objectives: To determine the half-life of haemofilters in critically ill patients admitted to the intensive care unit (ICU) and the main factors associated with their duration. 
Methodology: Cross-sectional observational descriptive study conducted in a polyvalent adult intensive care unit. The haemofilters placed in 67 patients over 18 years of age between January and November 2019 were studied. Variables: age, sex, weight, admission unit, blood flow velocity, filtration fraction, hourly debit, system anticoagulation, activated thromboplastin time (APTT), medical indication for therapy, cause of withdrawal, catheter location, start and end time of therapy.

Results: The mean age of the patients was 62.66 years $( \pm 9.95), 81(71.64 \%)$ men. A total of 238 haemofilters with a mean lifetime of 26.28 hours $( \pm 22.8$ ) were analysed. Femoral catheters accounted for 80.1 $\%$, jugular catheters for $19 \%$ and subclavian catheters for $0.8 \%$. Sodium heparin was used as anticoagulation therapy in $45.8 \%$, citrates in $20.2 \%$ and no anticoagulation in $34 \%$. Mean blood flow velocity was $190.08 \mathrm{ml} / \mathrm{min}( \pm 53.48)$. A statistical relationship was found between the variables blood flow ( $r s=0.208 ; p=0.001)$, catheter location and haemofilter duration $(p=0.03)$.

Conclusions: The half-life of the haemofilter was 26 hours. Blood flow velocity and catheter location are factors that affect the duration of the haemofilter

KEYWORDS: haemofiltration; critical care; anticoagulants; vascular access device; blood flow velocity.

\section{Introducción}

Las Técnicas Continuas de Reemplazo Renal son aquellas técnicas que incluyen la circulación continua de sangre por circuitos extracorpóreos con el fin de depurarla, y forman parte del tratamiento y cuidado integral de pacientes con lesión renal aguda ${ }^{1}$. Algunos autores prefieren el término más genérico de Técnicas Continuas de Depuración Extracorpórea (TCDE), ya que existen algunas modalidades que no son necesariamente continuas y otras que no suplen una deficiente función del riñón ${ }^{2}$. En este artículo hablaremos en todo momento de TCDE.

Esas técnicas permiten realizar una eliminación lenta y continuada de ultrafiltrado, con buena tolerancia hemodinámica en pacientes críticos ${ }^{3}$.Además, tienen una mayor rentabilidad depurativa y una mayor seguridad para el paciente ${ }^{4,5}$. Actualmente las TCDE son veno-venosas, y las más utilizadas son la ultrafiltración, hemodiálisis, hemo-filtración, hemodialfiltración y diálisis continua de alto flujo ${ }^{6}$. En nuestra UCI, mayoritariamente utilizamos la hemodiafiltración vena-venosa continua (HDFVVC); es una técnica asociada con ratios elevados de ultrafiltración y difusión a través de una membrana altamente permeable ${ }^{5}$.

Existen estudios que recomiendan cambios electivos del hemofiltro según la patología a tratar; a las $12 \mathrm{~h}$ en pacientes sépticos, a las $24 \mathrm{~h}$ cuando el objetivo es realizar depuración renal, y a las 72 h para la eliminación de líquido ${ }^{7}$. Pero a veces la teoría y la práctica no siempre confluyen por igual. Los estudios que han analizado la vida media de hemofiltros son antiguos y la media de duración descrita es variable ${ }^{7}$. La duración del hemofiltro y los factores que pueden influir, son de vital importancia dado los costes que puede ocasionar el correcto manejo del hemofiltro por parte del profesional. La aparición y desarrollo del dialytrauma, engloba todos los eventos adversos dañinos relacionados con la TCDE, cómo son las alteraciones metabólicas, hidroelectrolíticas y/0 terapéuticas ${ }^{8,9}$. Pueden surgir problemas derivados del propio hemofiltro o complicaciones derivadas de la clínica. Las complicaciones derivadas de la técnica son: la obstrucción y/o acodamiento del catéter, el calibre y localización de los accesos vasculares, la pauta de anticoagulación, la velocidad de flujo sanguíneo, el volumen de reemplazo de fluidos y electrolitos (descritos como factores que influyen en la vida útil del hemofiltro) ${ }^{10-12}$. De hecho, una de las complicaciones más frecuentes durante las TCDE es la coagulación del filtro, que se describe como una caída de presión superior a $100 \mathrm{mmHg}$ y una presión transmembrana (PTM) superior a $200 \mathrm{mmHg}$, de forma mantenida, lo que obliga a cambiar de manera precoz el sistema, sin cumplir a veces el tiempo de permanencia establecido por el fabricante, que son a las 72 horas $^{13}$. Las complicaciones derivadas de la propia clínica son la hemorragia al canalizar el acceso vascular, alteraciones del balance hídrico, hipotermia, trombosis y anemia por sustituciones repetidas de hemofiltros ${ }^{6,7}$. La coagulación del hemofiltro conlleva la pérdida hemática subyacente al no retorno del volumen sanguíneo remanente del sistema ${ }^{5}$. Se ha estimado que la pérdida de sangre cada vez que se coagula el hemofiltro M100 PRESET $^{\oplus}$ sin posibilidad de retorno, es de $150 \mathrm{ml}$, cantidad que varía según modelo de máquina y tamaño del mismo, lo que podría favorecer la aparición de anemia iatrogénica ${ }^{14,15}$. El cambio frecuente de los circuitos (hemofiltro y sistemas) interrumpe el tratamiento, supone un aumento de las cargas de trabajo, pueden aumentar el coste económico y ser perjudiciales para la atención al paciente ${ }^{16}$. 
A pesar de los factores descritos en la bibliografía que pueden influir en la optimización de la vida útil del hemofiltro, continúa siendo un aspecto de vital importancia realizar un diagnóstico de situación del entorno donde trabajamos para detectar aspectos de mejora en el cuidado de pacientes que precisan TCDE. Conocer las causas permitirá prevenir, lo que aumentará la duración de los equipos, reducirá costos económicos, optimizará la carga de trabajo de los profesionales de enfermería y permitirá aplicar estrategias de mejora en la atención y cuidado de pacientes portadores de hemofiltro.

Los objetivos de este estudio fueron determinar la vida media de los hemofiltros en el paciente crítico ingresado en la UCI y los principales factores asociados a su duración.

\section{Material y Método}

Diseño: Estudio descriptivo observacional transversal, realizado en la Unidad de Cuidados Intensivos (UCI) de un hospital público terciario, que dispone de 32 camas, distribuidas en 4 unidades: cirugía cardiaca, coronarias, médico quirúrgica y neurotraumatología. La recogida de datos se realizó desde enero hasta noviembre de 2019.

Población y muestra: La población a estudio fueron todos los hemofiltros utilizados en pacientes mayores de 18 años ingresados en la UCI. El servicio de Medicina Intensiva del Hospital Son Espases dispone de un consentimiento informado para el registro y uso de un conjunto mínimo de datos del paciente, obtenidos a partir de la historia clínica y gráficas evolutivas, y que es solicitado al paciente o familia al ingreso en la unidad. Este consentimiento fue evaluado favorablemente por el Comité de Ética de la Investigación de las Islas Baleares en sesión del día 27 de junio de 2019, referencia IB 3909/19 PI.

Variables: En nuestra unidad utilizamos el monitor de hemofiltración PrismaFlex ${ }^{\circledR}$, circuitos M100 y M150 PRESET ${ }^{\circledR}$ con membrana AN69, y 0 xiris $^{\circledR}$ de Hospal $^{\circledR}$. Se recogieron variables sociodemográficas al inicio de la terapia: edad, sexo, índice de masa corporal (IMC) y peso, unidad de ingreso, velocidad de flujo sanguíneo $(\mathrm{ml} / \mathrm{h})$, fracción de filtrado (FF), débito horario, terapia de anticoagulación utilizada para el sistema: heparina sódica (Heparina Sódica Sala ${ }^{\circledR} 5000 \mathrm{UI} / \mathrm{ml}$ ), citratos (Prismocitrate ${ }^{\circledR}$ ) o sin terapia de anticoagulación, tiempo de tromboplastina activada (TTPA), motivo de la retirada (coagulación del hemofiltro, problemas con el catéter, recambio del circuito por caducidad), hora de inicio y finalización de la terapia.

El catéter utilizado para las TCDE es GamCath ${ }^{\circledR}$ Gambro de doble luz de $11,5 \mathrm{Fr}$ y $150 \mathrm{~mm}$ para yugular, de $13 \mathrm{Fr}$ y $200 \mathrm{~mm}$ para femoral y de $13 \mathrm{Fr}$ y $150 \mathrm{~mm}$ para subclavia.

La administración de heparina sódica se realiza a través del hemofiltro en perfusión continua, a una velocidad $2,1 \mathrm{ml} / \mathrm{h}$ y dosis de heparina ajustada al peso del paciente. La dosis de citrato objetivo son de 2 a 5 $\mathrm{mmol} / \mathrm{l}$ de sangre. La velocidad de flujo de citrato se ajusta en proporción correcta con la velocidad de flujo sanguíneo.

Recogida de datos: El proceso de recogida de datos se realizó mediante una hoja auto diseñada. La cumplimentación de los registros fue llevada a cabo por los profesionales de la unidad y los investigadores, al inicio y finalización de la terapia. Posteriormente fueron transcritos a una base de datos.

Análisis estadístico: Se realizó un análisis descriptivo para las variables cuantitativas: medidas de tendencia central (media y mediana) y de dispersión (desviación estándar y rango intercuartílico). Para las variables cualitativas se calculó medidas de frecuencias y porcentajes. Para el análisis de las variables se utilizó la pruebas de Kruskal-Wallis y el coeficiente de correlación Rho de Spearman. Se consideró significación estadística valores de $p<0,05$. El análisis estadístico se realizó con el software IBM SPSS vs. 24.

Consideraciones éticas: El estudio fue presentado y aprobado por el comité de investigación del hospital el 14 de diciembre de 2018, referencia $n^{\circ}$ CI-286-18. Se ha garantizado la confidencialidad en todo momento siguiendo la Ley Orgánica de Protección de Datos y Garantía de Derechos Digitales (LOPDGDD) 3/2018 de 6 de diciembre. Los autores declaran no tener conflictos de intereses.

\section{Resultados}

Se recogieron datos de 238 hemofiltros en una muestra de 67 pacientes. Se descartaron otros tres hemofiltros por problemas en el montaje/cebado. La edad media de los pacientes fue de 62,66 años $( \pm 9,95)$, el $80,1 \%$ fueron hombres. En la tabla 1 se representan las características de los pacientes y unidad de ingreso. 
Tabla 1. Características de los pacientes y unidad de ingreso. Hemodiafiltración venovenosa continua (HDFVVC).

\begin{tabular}{|c|c|c|c|c|}
\hline & Cirugía cardiaca & Coronarias & Médico-quirúrgica & Neurotraumatología \\
\hline Hombres $(n=48)$ & $13(92,86 \%)$ & $9(64,29 \%)$ & $23(63,89 \%)$ & $3(100 \%)$ \\
\hline Mujeres $(n=19)$ & $1(7,14 \%)$ & $5(35,71 \%)$ & $13(36,11 \%)$ & ---- \\
\hline Edad (años) & $64,58( \pm 11,74)$ & $65,64( \pm 9,18)$ & $61,35( \pm 9,09)$ & $56,22( \pm 6,22)$ \\
\hline Peso $(\mathrm{Kg})$ & $102,96( \pm 26,5)$ & $101,92( \pm 16,24)$ & $85,59( \pm 17,23)$ & $113,83( \pm 21,94)$ \\
\hline IMC $\left(\mathrm{Kg} / \mathrm{m}^{2}\right)$ & $35,01( \pm 5,94)$ & $36,28( \pm 5,21)$ & $29,72( \pm 6,34)$ & $37,03( \pm 7,14)$ \\
\hline $\mathrm{N}^{0}$ hemofiltros $(\mathrm{n}=238)$ & 59 & 39 & 131 & 9 \\
\hline
\end{tabular}

Tabla 2. Duración de filtros en relación con la localización y número de catéteres.

\begin{tabular}{|l|c|c|c|c|}
\hline Localización & Flujos $\mathrm{ml} / \mathrm{min}$ & Duración Horas & \% sobre el total de catéteres & p \\
\hline Femoral $(\mathbf{n}: 189)$ & $193,39( \pm 52,98)$ & $28,37( \pm 22,93)$ & 80,2 & 0,99 \\
\hline Yugular $(\mathbf{n}: 45)$ & $178,67( \pm 53,62)$ & $20,34( \pm 21,33)$ & 19 & 0,03 \\
\hline Subclavia $(\mathbf{n}: 2)$ & $175( \pm 106,07)$ & $12( \pm 9,88)$ & 0,8 & no se puede calcular \\
\hline
\end{tabular}

En relación al abordaje de accesos vasculares, 189 $(80,1 \%)$ se insertaron en vena femoral, con un flujo medio de $193,39 \mathrm{ml} / \mathrm{min}( \pm 52,98) ; 46(19 \%)$ en vena yugular interna con flujo medio de $178,67 \mathrm{ml} / \mathrm{min}( \pm 53,62)$, y $2(0,8 \%)$ en la vena subclavia, con flujo medio de 175 $\mathrm{ml} / \mathrm{min}( \pm 106,07)$ (tabla 2).

Se calculó una media de 3,57 filtros $( \pm 5,95)$ por paciente. Según la indicación médica para iniciar tratamiento de TCDE, $154(63,5 \%)$ fue por insuficiencia renal, 45 (18,5\%) por shock séptico, $16(6,6 \%)$ sobrecarga hídrica, $13(5,4 \%)$ por alcalosis/acidosis metabólica, $4(1,66 \%)$ por shock distributivo, 3 (1,24\%) por hiperpotasemia, $2(0,8 \%)$ por depuración por intoxicación, $2(0,8 \%)$ en postoperatorio de cirugía cardiaca y $3(1,24 \%)$ por otras patologías.

La velocidad media de flujo sanguíneo (VMFS) fue de $190,08 \mathrm{ml} / \mathrm{min}( \pm 53,48)$, con una FF media (cantidad de suero que ultrafiltramos del total de plasma que pasa por el filtro en un determinado período de tiempo) de $19,02( \pm 7,45)$, y vida media del filtro de 26,28 horas $( \pm 22,8)$.

En relación con la terapia de anticoagulación, se empleó la heparina sódica en $105(44,12 \%)$ de los hemofiltros, en $48(20,17 \%)$ se utilizó citratos, y en $81(34,03 \%)$ no se utilizó anticoagulación por contraindicación médica (presencia o riesgo aumentado de sangrado, alteraciones de la coagulación, uso de anticoagulantes sistémicos, plaquetopenia, trombocitopenia inducida por heparina). En uno de los hemofiltros $(0,42 \%)$ la heparina fue aportada junto a la terapia ECMO y en $3(1,26 \%)$ la heparina se suministró a través del catéter central. Las dosis medias de heparina pautada fueron de 5,60 U/kg/h $( \pm 2,88)$. El valor medio de TTPA del paciente se mantuvo de media en $37,69( \pm 13,5)$ segundos.

En relación a los factores que motivaron la interrupción de la terapia, en $128(53,9 \%)$ de los casos la causa fue la coagulación del hemofiltro con una duración media de este de $22,72( \pm 16,82)$ horas, en $32(13,3 \%)$ de los casos el hemofiltro llegó al final de su vida útil con una duración media de estos de $63,23( \pm 23,72)$ horas y, en otros $32(13,3 \%)$ la causa fue problemas con el catéter y su duración media fue de 11,97 horas $( \pm 7,60)$.

Por problemas relacionados con el acceso vascular (presiones, obstrucción, acodamientos...) fueron retirados y sustituidos $14(18,8 \%)$ de los circuitos tratados con citratos, $23(11,4 \%)$ en los de heparina y $9(11,1 \%)$ en los que no llevaron anticoagulación.

En relación con la asociación de variables, no se encontraron diferencias estadísticamente significativas entre la durabilidad de los hemofiltros sin anticoagulación, los anticoagulados con heparina y citratos $(p=0,6)$ (figura 1 ). 


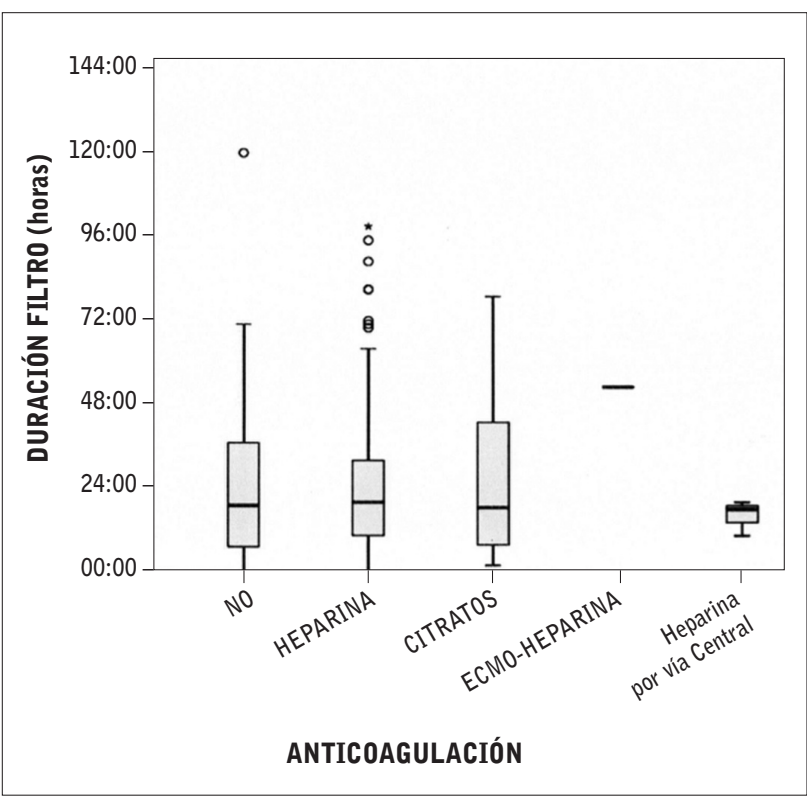

Figura 1. Anticoagulación utilizada y duración del hemofiltro.

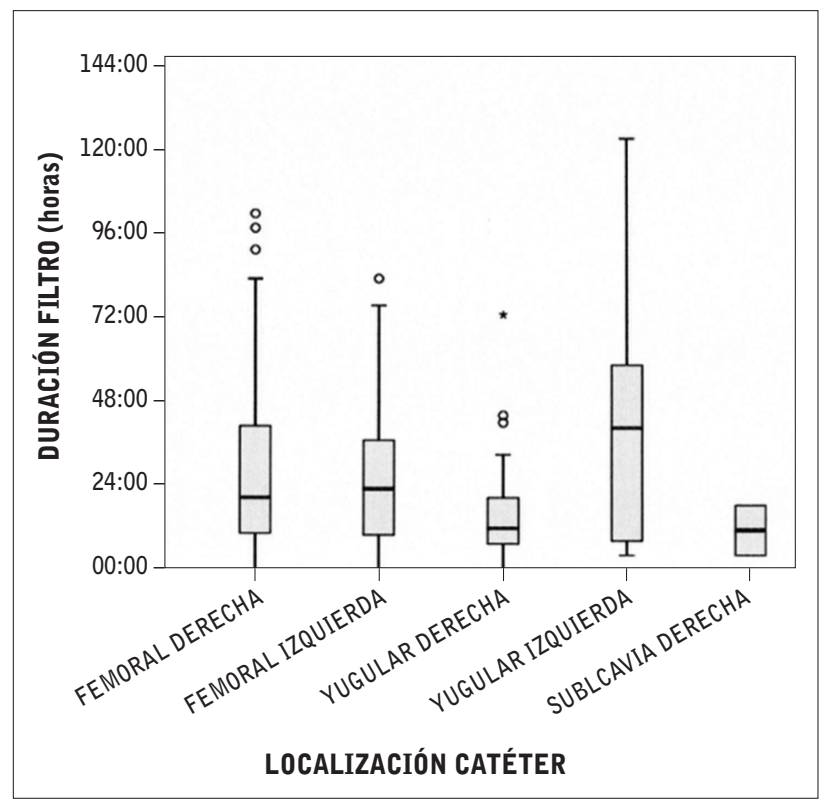

Figura 2. Localización del catéter y duración del hemofiltro.

Se encontró relación estadística entre la velocidad del flujo sanguíneo y la durabilidad del hemofiltro ( $r s=0,208 ; p=0,001$ ); flujos más altos tuvieron duraciones más elevadas del hemofiltro. Los hemofiltros con catéteres localizados en vena yugular izquierda perduraron más horas que los de localización femoral y subclavia (figura 2). En la tabla 2 se representa la duración del hemofiltro respecto a la localización de los distintos catéteres.
No se encontró relación estadística entre la vida media del hemofiltro y los valores de TTPA $(r=0,037, p=0,6$.). En la tabla 3 están representados el anticoagulante utilizado, VMFS, FF, pérdida horaria y duración del hemofiltro.

Posterior a determinar la relación entre las variables, se aplicó el análisis de regresión lineal múltiple para determinar la relación entre aquellas que podían influir en la duración del hemofiltro. La variable flujo de sangre es un determinante que se asoció a la duración del filtro (tabla 4).

\section{Discusión}

La utilización de las TCDE en el paciente crítico y los continuos avances en este tipo de terapias tanto en los materiales como en los dispositivos, han permitido ampliar las indicaciones de las TCDE ${ }^{17}$. Una de las principales ventajas de la HDFVVC es que permite su utilización en pacientes inestables ya que es un tratamiento lento y continuado, que evita cambios bruscos en la volemia y permite una gran flexibilidad en el manejo hidroelectrolítico y el control metabólico ${ }^{5}$.

En nuestro servicio durante la realización de este estudio solo se utilizó la hemodiafiltración como terapia de TCDE, esta modalidad se utiliza en varios de los estudios consultados ${ }^{10,18-21}$. La localización del acceso vasculares es una variable a tener en cuenta en la planificación de la técnica por su relevancia sobre la presencia de complicaciones. La diversidad de catéteres disponibles en función del punto de inserción proporciona los flujos de sangre necesarios para la terapia ${ }^{10}$. El sitio de elección para la inserción del catéter temporal para la terapia es la vena yugular interna derecha, debido a que el recorrido intravascular es recto y existe menor riesgo de trombosis ${ }^{6,22}$. Así pues, el tipo de acceso vascular y el lugar de inserción son elementos a tener en cuenta para un óptimo funcionamiento del circuito, ya que para poder realizar TCDE es necesario disponer de un acceso que proporcione un buen flujo de sangre y baja resistencia al retorno ${ }^{23}$. En nuestro estudio, se registró un mayor número de catéteres insertados en la vena femoral. Es uno de los accesos de elección en pacientes críticos con escasa movilización, porque resulta fácil de abordar y es bastante segura ${ }^{24}$. Se observó una asociación entre el número de hemofiltros coagulados y dicha localización, siendo este resultado similar a otros estudios consultados ${ }^{10,20,25}$. A pesar de que hay un mayor número de catéteres femorales, según el análi- 
Tabla 3. Anticoagulación utilizada, vida media de los hemofiltros utilizados, velocidad media de flujo sanguíneo (VMFS), fracción de filtrado (FF).

\begin{tabular}{|c|c|c|c|c|c|}
\hline & $\mathbf{P}$ & & $\begin{array}{c}\text { Sin } \\
\text { anticoagulación }\end{array}$ & Heparina & Citratos \\
\hline \multirow{6}{*}{$\begin{array}{l}\text { Duración } \\
\text { media } \\
\text { hemofiltros } \\
\text { (horas) }\end{array}$} & \multirow{6}{*}{0,6} & Media & 25,42 & 26,35 & 27,47 \\
\hline & & Mediana & 20 & 21 & 19 \\
\hline & & Desviación estándar & $( \pm 23,32)$ & $( \pm 22,30)$ & $( \pm 23,33)$ \\
\hline & & Rango & 121 & 100 & 80 \\
\hline & & Máximo & 121 & 100 & 80 \\
\hline & & Mínimo & 0 & 0 & 0 \\
\hline \multirow{6}{*}{$\begin{array}{l}\text { VMFS } \\
(\mathrm{ml} / \mathrm{min})\end{array}$} & \multirow{6}{*}{0} & Media & 192,84 & 210,57 & 144,42 \\
\hline & & Mediana & 200 & 200 & 135 \\
\hline & & Desviación estándar & $( \pm 54,64)$ & $( \pm 45,35)$ & $( \pm 37,39)$ \\
\hline & & Rango & 230 & 220 & 200 \\
\hline & & Máximo & 300 & 300 & 300 \\
\hline & & Mínimo & 70 & 80 & 100 \\
\hline \multirow{6}{*}{ FF (\%) } & \multirow{6}{*}{0,3} & Media & 14,93 & 17,27 & 28,57 \\
\hline & & Mediana & 16 & 17 & 30 \\
\hline & & Desviación estándar & $( \pm 5,2)$ & $( \pm 5,14)$ & $( \pm 5,88)$ \\
\hline & & Rango & 33 & 33 & 29 \\
\hline & & Máximo & 36 & 35 & 37 \\
\hline & & Mínimo & 3 & 2 & 8 \\
\hline \multirow{6}{*}{$\begin{array}{l}\text { Pérdida } \\
\text { horaria } \\
(\mathrm{ml} / \mathrm{h})\end{array}$} & \multirow{6}{*}{0,1} & Media & 93,46 & 128,29 & 86,15 \\
\hline & & Mediana & 50 & 150 & 100 \\
\hline & & Desviación estándar & $( \pm 108,08)$ & $( \pm 89,70)$ & $( \pm 85,80)$ \\
\hline & & Rango & 350 & 300 & 300 \\
\hline & & Máximo & 350 & 300 & 300 \\
\hline & & Mínimo & 0 & 0 & 0 \\
\hline
\end{tabular}

VMFS: Velocidad media del flujo de sangre. sis los de mayor durabilidad fueron los insertados en yugular izquierda. En una revisión bibliográfica reciente, se recomienda utilizar catéteres de acceso yugular debido a la anatomía del vaso, ya que es más corto y recto, por lo que favorecen la optimización y rendimiento del hemofiltro ${ }^{26}$. Un aspecto en el uso de las TCDE es la variación de la velocidad del flujo sanguíneo en función del anticoagulante utilizado. En nuestro estudio las velocidades oscilaron de 110 a $225 \mathrm{ml} / \mathrm{min}$. La bibliografía encontrada, describe una velocidad media de flujo que oscila entre $100 \mathrm{ml} / \mathrm{min}$ y 300 $\mathrm{ml} / \mathrm{min}$, donde la modalidad principal utilizada es la HDFVVC, lo que indica una gran variabilidad para estos tratamientos $10,19-21,25,27,28$. Cabe destacar que los hemofiltros con mayor durabilidad se habían asociado a una mayor velocidad del flujo sanguíneo. En el estudio de Mottes se calculó que por cada 10 $\mathrm{ml} / \mathrm{min}$ de aumento de flujo sanguíneo, equivale a un aumento del $5,8 \%$ en la supervivencia del hemofiltro ${ }^{29}$.

El número así como la vida media de los hemofiltros difieren de otras series consultadas, aunque próximos a los márgenes descritos en estos estudios $(3,57$ frente a 4,4 y 6,8 filtros por paciente, con una duración media de 26,28 horas frente a 17,72 y 49,4 horas $)^{7,18}$. En nuestro estudio no observamos diferencias significativas entre la duración del hemofiltro con anticoagulación y sin anticoagulación. En los estudios consultados, la vida media oscila entre 18,7 y $49,9 \mathrm{~h}$, y en hemofiltro con citratos llegan hasta las 92,8 $h^{7,16}$.

Tabla 4. Análisis de regresión lineal múltiple. Variable dependiente: Duración del filtro.

\begin{tabular}{l|c|c|c|c|c|}
\hline & \multicolumn{2}{|c|}{ Coeficientes no estandarizados } & Coeficientes estandarizados & & t \\
\hline Determinantes & Beta & Error estándar & Beta estandarizada & $-1,742$ & 0,083 \\
\hline Localización del catéter & $-10250,6$ & 5885,372 & $-0,113$ & 2,090 & 0,038 \\
\hline Flujo de sangre & 212,2 & 101,550 & 0,138 & 1,045 & 0,297 \\
\hline Anticoagulación & 6991,3 & 6690,05 & 0,069 & & p \\
\hline
\end{tabular}


Cabría esperar que a valores más elevados de TTPA, la vida media del hemofiltro fuese mayor. Según el estudio de Bouman, existe una asociación positiva entre el TTPA y la vida media de los hemofiltros ${ }^{30}$. Nuestros datos reflejaron que valores más bajos de TTPA coincidieron con hemofiltros de vida media más corta, pero no se ha podido demostrar estadísticamente.

La coagulación del hemofiltro implica la retirada de la terapia o el recambio del circuito y continuar con la terapia. Existen estudios que afirman que la coagulación es una de las principales causas de retirada $(22,6 \%$; $29,6 \%)^{7,10}$, siendo en nuestro estudio muy superior $(53,9 \%)$. Entre los factores asociados a la coagulación del hemofiltro, el mal funcionamiento del catéter puede ser un factor de riesgo de coagulación del hemofiltro ${ }^{31}$ nuestros resultados también son superiores a los encontrados en la bibliografía $(13,3 \% \text { vs } 5,8 \%)^{32}$.

Para prolongar la vida media de los hemofiltros es necesario una monitorización constante de las presiones del circuito (especialmente la tendencia, que proporciona información sobre el estado del sistema), utilización de catéteres apropiados, modificación de la fracción de filtración para mantenerla por debajo del $20 \%$ o realizar $1 / 3$ de la reposición prefiltro ${ }^{6}$. El número de alarmas, los problemas mecánicos que puedan surgir durante la terapia y la falta de formación se han asociado a peor vida útil del hemofiltro $0^{9,33}$. En un estudio realizado en nuestra unidad sobre formación en TCDE, las enfermeras y médicos manifestaron la necesidad de formación y reciclaje continuo $0^{34,35}$. A pesar de haber realizado formación, la incorporación de nuevo personal, y una continua rotación de parte de los profesionales de enfermería, hacen que la formación recibida quede obsoleta a corto-medio plazo ${ }^{34}$. Una auditoría de la vida útil del hemofiltro es una información útil y necesaria para las estrategias de formación del personal de enfermería. Quizás se debería insistir en realizar más formación en el control de la técnica y conocimiento de aquellas variables que puedan predecir y detectar una coagulación precoz. La coagulación del hemofiltro es un evento adverso $0^{36}$ por lo que fomentar una actitud proactiva y promover estrategias para prevenir riesgos asociados a la terapia, puede mejorar la eficacia de la terapia y mejorar la seguridad del paciente.

Como limitaciones del estudio, hay que destacar el tamaño muestral, al tratarse de un estudio realizado en un único centro. En nuestra UCI, a pesar de disponer de un protocolo de anticoagulación con citratos, existe mucha variabilidad a la hora de prescribir la terapia de anticoagulación, no existiendo un consenso cuando se prescribe una terapia con otro anticoagulante, como por ejemplo la heparina sódica. Aún siendo referencia en el campo de las TCDE, la obsolescencia de algunas de las publicaciones científicas consultadas han dificultado poder comparar los resultados con estudios más actuales. A partir de los resultados obtenidos en nuestro estudio podemos apreciar que la vida media de los hemofiltros estudiados está en torno a 26 h, situándose dentro de los rangos descritos por otros autores. Los hemofiltros sin anticoagulación presentan valores similares en cuanto a duración y velocidad media respecto a los hemofiltros con anticoagulación. Los hemofiltros en los que el acceso vascular era un catéter yugular izquierdo registraron mayor número de horas de supervivencia respecto al resto de localizaciones.

Debemos plantearnos mejorar el tiempo de duración de los hemofiltros e invertir esfuerzos en mejorar la formación de enfermeras y médicos, para reducir riesgos y costes asociados a las TCDE.

Recepción: 28-02-21

Aceptación: 26-10-21

Publicación: 30-12-21

\section{Bibliografía}

1. Bagshaw SM, George C, Bellomo R, ANZICS Database Management Committe. A comparison of the RIFLE and AKIN criteria for acute kidney injury in critically ill patients. Nephrol Dial Transplant Off Publ Eur Dial Transpl Assoc - Eur Ren Assoc. 2008 May;23(5):1569-74.

2. Úbeda-Iglesias A, Herrera-Rojas D, Gómez-González C. Encuesta sobre el manejo del fracaso renal agudo y las técnicas de reemplazo renal en las unidades de cuidados intensivos españolas. Med Intensiva. 2015;39(2):84-9.

3. Herrera Gutiérrez ME. Técnicas de reemplazo renal continuas frente a las intermitentes: pro-continuas. Medicina Intensiva. 2009;33(2):88-92. 
4. Rodríguez Segura MD, Moreno Vicente $M$, Pérez Rodríguez N. Técnicas continuas de depuración extrarrenal en el paciente crítico. Enferm Integral. 2015;108:39-41.

5. Gutiérrez Rodríguez P. Técnicas continuas de depuración renal extracorpórea en el paciente crítico: fundamentos y componentes del sistema de terapia. Tiempos de enfermería y salud. 2020;2(9):14-9.

6. Romero-García M, de la Cueva-Ariza L, Delgado-Hito P. Actualización en técnicas continuas de reemplazo renal. Enferm Intensiva. 2013 Jul;24(3):113-9.

7. García Olert A, Hernández Sánchez AI, Miralles Andujar FJ, Cortés Carmona J, Domínguez Bernal MÁ, Caro Nieto ME. Experiencia en las técnicas continuas de reemplazo renal en cuidados intensivos: Determinantes de la duración del hemofiltro. Rev Soc Esp Enferm Nefrológica. 2008;11:259-64.

8. Guirao Moya A, Esteban Sánchez M, Fernández Gaute N, Murga González A., Vergara Diez L., Martínez García M.P., et al. Monitorización de presiones en técnicas continuas de depuración extrarrenal. Enferm Intensiva. 2010;21(1):28-33.

9. Maynar Moliner J, Honore PM, Sánchez-Izquierdo Riera JA, Herrera Gutiérrez M, Spapen HD. Handling continuous renal replacement therapy-related adverse effects in intensive care unit patients: the dialytrauma concept. Blood Purif. 2012;34(2):17785.

10. Molano Álvarez E, Guirao Moya A, Esteban Sánchez $M E$, Sanz Cruz P, García Delgado E, Sánchez-Izquierdo Riera JÁ. Detección de riesgos para la seguridad del paciente en la implementación de técnicas continuas de depuración extracorpórea. Enferm Intensiva. 2011;22(1):39-45.

11. Molano Álvarez E, Cornejo Bauer C, García Hernández R, Rojo Cabello $S$, Cuenca Solanas $M$, García Fuentes C. Enfermería de cuidados críticos y técnicas continuas de reemplazo renal en la Comunidad de Madrid. Enferm Intensiva. 2003;14(4):135-47.

12. Sanz M, Hidalgo F, García-Fernández N. Supervivencia de circuitos de técnicas de depuración extrarrenal continua en pacientes críticos con o sin anticoagulación convencional: estudio observacional prospectivo. An Sist Sanit Navar. 2017;40(1):77-84.
13. Gaínza FJ, Sánchez-Izquierdo JA, Poch E, Maduell F, Solozábal C, Otero A, et al. Guías SEN - Actuación en el fracaso renal agudo. Tratamiento sustitutivo de la función renal. Nefrol 2007; 27(Supl 3):109-91.

14. Shoemaker W, Ayes $S$, Grenvik A, Holbrook P. Shoemaker W, Ayes S, Grenvik A, Holbrook P. Tratado de Medicina Crítica y Terapia Intensiva. Médica Panamericana. Madrid: Médica Panamericana; 4.a ed; 2002 .p. 1673.

15. Vinagre Gaspar R, Cornejo Bauer C, Murillo Pérez A, Molano Alvarez E, Muñoz López 0, Morales Sánchez $C$, et al. Pérdidas sanguíneas diagnósticas en pacientes con trauma grave. Enferm Intensiva. 2010 Sep;21(3):120-5.

16. Houllé-Veyssière $M$, Courtin $A$, Zeroual N, Gaudard $\mathrm{P}, \mathrm{C}$ Colson $\mathrm{PH}$. Continuous venovenous renal replacement therapy in critically ill patients: A work load analysis. Intensive Crit Care Nurs. 2016t;36:35-41.

17. Reyes-Marín FA. Hemodiálisis y terapias continuas. Gac Méd Méx. 2008;144(6):517-19.

18. Herrera Gutiérrez $M E$, Daga Ruiz $D$, Seller Pérez G, García Alcántara A, De la Rubia de Gracia C, Ruiz del Fresno $L$. Uso de las técnicas continuas de reemplazo renal en las Unidades de Cuidados Intensivos en España. Una encuesta a nivel nacional. Med Intensiva. 2000;24(8):341-7.

19. Navas A, Ferrer $R$, Martínez $M$, Martínez ML, Haro C de, Artigas A. Terapia de reemplazo renal en paciente crítico: cambios evolutivos del tratamiento en los últimos años. Med Intensiva. 2012;36(8):540-7.

20. Tomasa Irriguible TM, Sabater Riera J, Poch Lopez de Briñas L de, Fort Ros J, LLoret Cora MJ, Roc Antònio J, et al. Manejo actual de las terapias continuas de reemplazo renal: Estudio epidemiológico multicéntrico. Med Intensiva. 2017;41(4):216-26.

21. Fealy N, Aitken L, Toit E du, Baldwin I. Continuous renal replacement therapy: current practice in Australian and New Zealand intensive care units. Crit Care Resusc J Australas Acad Crit Care Med. 2015 Jun;17(2):83-91. 
22. Echevarria-Uraga JJ, García-Garai N, Muñoz-González RI. Accesos venosos para hemodiálisis: abordaje yugular. Diálisis Traspl. 2014;35(4):163-8.

23. Cobo Sánchez JL, Menezo Viadero R, Gándara Revuelta M, Pelayo Alonso R, Villa Llamazares C, Maza Sota MA, et al. ¿Influye tanto el flujo de sangre en la eficacia dialítica?. Comunicaciones presentadas al XXXI Congreso Nacional de la Sociedad Española de Enfermería Nefrológica. 2008. p. 389-94.

24. Gallego Cobos N, Dorao Martínez-Romillo R. Fracaso renal agudo en el paciente pediátrico. Nefrología. 2007; 27(s3):S257-74.

25. Kee YK, Kim EJ, Park KS, Han SG, Han IM, Yoon CY, et al. The effect of specialized continuous renal replacement therapy team in acute kidney injury patients treatment. Yonsei Med J. 2015 May;56(3):658-65.

26. Juncos LA, Chandrashekar K, Karakala N, Baldwin I. Vascular access, membranes and circuit for CRRT. Semin Dial. 2021;00:1-10.

27. Oh HJ, Lee MJ, Kim CH, Kim DY, Lee HS, Park JT, et al. The benefit of specialized team approaches in patients with acute kidney injury undergoing continuous renal replacement therapy: propensity score matched analysis. Crit Care. 2014 Ago;18(4):454.

28. Carneiro $P$, Santiago MJ, López J, Castillo $A$, López-Herce J. Anticoagulación regional con citrato en las técnicas de depuración extrarrenal continuas. An Pediatr Barc Spain 2003. 2012 Ene;76(1):49-50.

29. Mottes $T$, Owens $T$, Niedner M, Juno J, Shanley TP, Heung M. Improving delivery of continuous renal replacement therapy: impact of a simulation-based educational intervention. Pediatr Crit Care Med J Soc Crit Care Med World Fed Pediatr Intensive Crit Care Soc. 2013 0ct;14(8):747-54.
30. Bouman CSC, de Pont A-CJM, Meijers JCM, Bakhtiari K, Roem D, Zeerleder S, et al. The effects of continuous venovenous hemofiltration on coagulation activation. Crit Care Lond Engl. 2006;10(5):R150.

31. Valdenebro M, Martín-Rodríguez L, Tarragón B, Sánchez-Briales $P$, Portolés J. Terapia de reemplazo renal en pacientes críticamente enfermos con lesión renal aguda: perspectiva del nefrólogo 2020. Nefrologia. 2021;41(2):102-14.

32. Brain $M$, Winson $E$, Roodenburg 0 , McNeil J. Non anti-coagulant factors associated with filter life in continuous renal replacement therapy (CRRT): a systematic review and meta-analysis. BMC Nephrol. 2017 Feb;18(1):69.

33. Kim IB, Fealy N, Baldwin I, Bellomo R. Premature circuit clotting due to likely mechanical failure during continuous renal replacement therapy. Blood Purif. 2010;30(2):79-83.

34. Berrocal FJ, Maqueda M, De Dios A, Moreno C, Jimenez J. Percepción de los conocimientos adquiridos en técnicas continuas de depuración extracorpórea en profesionales de cuidados intensivos. Tesela [Internet]. 2020 [consultado 04 Mar 2021];27(el2657). Disponible en: http://ciberindex.com/index.php/ts/ article/view/el2657.

35. Tobo Medina N. Evaluación de una intervención de Enfermería para prevenir la coagulación del circuito extracorpóreo en hemodiálisis. Enferm Glob. 2015;14:276-85.

36. Arenas Jiménez MD, Ferre G, Álvarez-Ude F. Estrategias para aumentar la seguridad del paciente en hemodiálisis: Aplicación del sistema de análisis modal de fallos y efectos (sistema AMFE). Nefrol Madr. 2017;37:608-21.

Este artículo se distribuye bajo una Licencia Creative Commons Atribución-NoComercial 4.0 Internacional. https://creativecommons.org/licenses/by-nc/4.0/

Open Access (C) (1) (8) 